OPEN ACCESS

Edited by:

Heriberto Rodriguez-Martinez, Linköping University, Sweden

Reviewed by:

Cinzia Allegrucci, University of Nottingham, UK

Amy C. Lossie,

National Institutes of Health, USA Carlos M. Guerrero-Bosagna, Linköping University, Sweden

*Correspondence: Kirsten C. Sadler

kirsten.edepli@nyu.edu

Specialty section: This article was submitted to Epigenomics and Epigenetics, a section of the journal Frontiers in Genetics

Received: 24 December 2015 Accepted: 31 May 2016

Published: 17 June 2016

Citation:

Zhang C, Hoshida Y and Sadler KC (2016) Comparative Epigenomic Profiling of the DNA Methylome in Mouse and Zebrafish Uncovers High Interspecies Divergence. Front. Genet. 7:110 doi: 10.3389/fgene.2016.00110

\section{Comparative Epigenomic Profiling of the DNA Methylome in Mouse and Zebrafish Uncovers High Interspecies Divergence}

\author{
Chi Zhang ${ }^{1,2,3}$, Yujin Hoshida ${ }^{1,3}$ and Kirsten C. Sadler ${ }^{1,2,3,4 *}$ \\ ${ }^{1}$ Department of Medicine/Division of Liver Diseases, Icahn School of Medicine at Mount Sinai, New York, NY, USA, \\ ${ }^{2}$ Department of Developmental and Regenerative Biology, Icahn School of Medicine at Mount Sinai, New York, NY, USA, \\ ${ }^{3}$ Liver Cancer Program/Tisch Cancer Institute, Icahn School of Medicine at Mount Sinai, New York, NY, USA, ${ }^{4}$ Program in \\ Biology, New York University Abu Dhabi, Abu Dhabi, UAE
}

The DNA methylation landscape is dynamically patterned during development and distinct methylation patterns distinguish healthy from diseased cells. However, whether tissue-specific methylation patterns are conserved across species is not known. We used comparative methylome analysis of base-resolution DNA methylation profiles from the liver and brain of mouse and zebrafish generated by reduced representation bisulfite sequencing to identify the conserved and divergent aspects of the methylome in these commonly used vertebrate model organisms. On average, 24\% of CpGs are methylated in mouse livers and the pattern of methylation was highly concordant among four male mice from two different strains. The same level of methylation (24.2\%) was identified in mouse brain. In striking contrast, zebrafish had 63 and 70\% of CpG methylation in the liver and brain, respectively. This is attributed, in part, to the higher percentage of the zebrafish genome occupied by transposable elements (52\% vs. $45 \%$ in mice). Thus, the species identity was more significant in determining methylome patterning than was the similarity in organ function. Conserved features of the methylome across tissues and species was the exclusion of methylation from promoters and from $\mathrm{CpG}$ islands near transcription start sites, and the clustering of methylated CpGs in gene bodies and intragenic regions. These data suggest that DNA methylation reflects species-specific genome structure, and supports the notion that DNA methylation in non-promoter regions may contribute to genome evolution.

Keywords: DNA methylation, comparative epigenomics, mouse, zebrafish, liver, brain

\section{INTRODUCTION}

Cytosine methylation is a major epigenetic mark in many species, as it serves to significantly alter the accessibility of CpG sites across the genome (Deaton and Bird, 2011). The methylome changes dramatically during development (Singer et al., 2014; Zhao et al., 2014), and there are marked differences in pattern of methylation across different cell types and in many pathologies, most notably, cancer. Indeed, many studies using model organisms demonstrate a required role for DNA methylation in regulating vertebrate development (Bird, 2002; Messerschmidt et al., 2014) 
and in causing cancer (Eden et al., 2003; Gaudet et al., 2003; Howard et al., 2008; Mudbhary et al., 2014). The canonical functions for DNA methylation are to promote genomic imprinting (Li et al., 1993), X-chromosome inactivation (Boumil and Lee, 2001), preservation of chromosome stability (Smith and Crocitto, 1999) and transposon repression (Slotkin and Martienssen, 2007; Zemach et al., 2010); these functions are largely conserved across taxa. However, not all species methylate their genomes, and those that do exhibit a wide range of DNA methylation levels and patterns (Lee et al., 2010; Zemach et al., 2010). Even when the same region of the brain is compared between closely related species (humans and chimpanzees), marked differences in methylation levels and the pattern of methylation have been reported (Zeng et al., 2012). Whether the species-specific methylome difference reflects functional differences played by DNA methylation in different organisms or, instead, whether they mirror, and perhaps contribute to, genome evolution remains an open and important question.

Many studies on DNA methylation are based on the hypothesis that methylation serves to repress gene expression (McGhee and Ginder, 1979). There are, however, many exceptions to this pattern, and, indeed, it is now widely accepted that differences in promoter methylation play a regulatory role in only a few well publicized cases. In contrast, overwhelming evidence shows that, across cell types and species, 1000s of silenced genes have entirely unmethylated promoters and, conversely, many highly expressed genes have high levels of promoter methylation (Bestor et al., 2015). While CpG islands (CpGi) exert regulatory function on gene expression, their protection from DNA methylation is a conserved feature of CpGis (Long et al., 2016). However, the picture is becoming more complex, as recent work has shown that enhancer methylation is correlated with gene expression in developing zebrafish and other vertebrates (Lee et al., 2015; Bogdanović et al., 2016) and other studies show that moderately expressed genes do not have a strong correlation with methylation differences at upstream regulatory regions, but instead these genes are highly methylated throughout the gene body (Elliott et al., 2015). Thus, the understanding of how DNA methylation impacts gene expression is evolving.

Comparative methylome analysis across taxa has uncovered widely divergent methylation patterns across species (Feng et al., 2010; Lee et al., 2010; Zemach et al., 2010), supporting the conclusion that methylation cannot be a universal mechanism of repressing gene expression, or, perhaps, that DNA methylation may serve different functions across the branches of the phylogenetic tree. In contrast, the high level of methylation of transposable elements (TEs) is a feature of DNA methylation that is conserved from plants to animals (Zemach et al., 2010). This is proposed to be a central mechanism to repress the potentially catastrophic activation of these endogenous parasites (Yoder et al., 1997). Comparative studies further supported the hypothesis that DNA methylation function is nuanced and variable. Moreover, the stark differences in DNA methylation between vertebrates and invertebrates limit studies on the functional consequences of methylome repatterning to vertebrate models such as mouse and zebrafish.
We reasoned that if DNA methylation is a central mechanism of regulating gene expression, then this should be reflected in conserved methylation patterns of genes that have similar expression patterns across species. The liver serves a fundamental role in metabolic homeostasis in all vertebrates, and this is reflected in a shared pattern of gene expression from humans to fish (Lam et al., 2006; Mudbhary et al., 2014). We selected this organ for comparative methylome analysis between mouse and zebrafish because there are striking similarities in liver cell composition and function (Goessling and Sadler, 2015). Studies in zebrafish also show that accurate DNA methylation is essential for liver development and regeneration (Sadler et al., 2007; Jacob et al., 2015) and that loss of methylation causes liver cancer (Mirbahai et al., 2011).

Multiple approaches have been developed and used to characterize genome-wide DNA methylation (Reid, 2010). Of these, reduced representation bisulfite sequencing (RRBS) is a cost-efficient method to survey $\mathrm{CpG}$ methylation genome wide by sampling regions of the genome that are $\mathrm{CpG}$ rich for bisulfite sequencing (Gu et al., 2011). A drawback to this approach is that it mainly covers $\mathrm{CpG}$ islands at the expense of other genomic regions, In order to represent more $\mathrm{CpG}$ sites and increase the coverage of all genomic regions interrogated, a recently published enhanced RRBS (ERRBS; Akalin et al., 2012; GarrettBakelman et al., 2015) allows greater distribution across the genome of the mapped reads from bisulfite converted genomic DNA. Hepatic function and the population of hepatic cells is similar between zebrafish and mammals (Goessling and Sadler, 2015) and we asked whether these similarities would be reflected by conserved features of the methylome. We used ERRBS to generate single-nucleotide resolution DNA methylation map of the mouse liver and compared this to a previously described methylome analysis of zebrafish liver generated by RRBS. We report marked interspecies differences in methylation patterns, and by comparing the methylation landscape between liver and brain of mouse and zebrafish, we found that the difference between species is much more significant than differences between tissues of the same species. Our findings suggest that genome structure is the primarily determinant of methylome patterning.

\section{MATERIALS AND METHODS}

\section{Sample Preparation}

The four mouse samples (Mixed-1, Mixed-2, B6-1, and B6-2) that were used for bisulfite sequencing are all males and are from two different genetic backgrounds: B6-1 and B6-2 are C57BL/6 mice, whereas the mixed mice are on an undefined background including $\mathrm{C} 57 \mathrm{BL} / 6 \mathrm{~J}$ and other strains. Total livers were isolated from them between 6 and 8 weeks old and frozen at $-80^{\circ} \mathrm{C}$ for DNA isolation. Genomic DNA was extracted from each sample using the Qiagen DNA isolation kit according to the manufacturer's instruction. RNA was purified using Qiagen RNAeasy columns. The animal work was approved by Institutional Animal Care and Use Committee (IACUC) at the Brigham and Women's Hospital (mixed samples) 
and the Memorial Sloan Kettering Cancer Center (C57BL/6 samples).

\section{Enhanced Reduced Representation Bisulfite Sequencing (ERRBS) Library Preparation and Sequencing}

Bisulfite-converted DNA sequencing libraries were generated for each of the studied samples, which measure both $5 \mathrm{hmC}$ and $5 \mathrm{mC}$ in methylated fraction and $5 \mathrm{fC}, 5 \mathrm{caC}$, and $\mathrm{C}$ in unmethylated fraction. In brief, $50 \mathrm{ng}$ of high quality genomic DNA in $50 \mu \mathrm{l}$ of DNase-free water was used as starting material. The whole library preparation includes enzyme digestion of genomic DNA which enriched CpG rich regions, Phenol:chloroform clean up, End-repair, A-tailing, adapter ligation, size selection, bisulfite conversion, enrichment PCR, and quality control. Experimental details can be referred to the published ERRBS protocol (Smith et al., 2009; Garrett-Bakelman et al., 2015). These amplified libraries were sequenced on the Hiseq2000 platform for 50 cycles single end read runs at the Epigenomics Core facility in the Department of Medicine, Weill Cornell Medical College (New York, NY, USA). Image capture, analysis and base calling were performed using Illumina's CASAVA 1.8. Data is available in GEO, accession numbers are GSM2136660, GSM2136661, GSM2136662, and GSM2136663.

\section{RNA Sequencing Library Preparation and Sequencing}

For each of the mouse samples, Mixed-1, Mixed-2 and an additional mixed background age-matched mouse from the Brigham and Women's facility (Mixed-3), RNAseq libraries were prepared according to Illumina's TruSeq RNA sample preparation version 2 protocol. The 3 samples were sequenced using Illumina's HiSeq 2500 platform (100bp paired-end sequencing) generating $\sim 64$ million reads, $\sim 60$ million reads and $\sim 53$ million reads for samples Mixed-1, Mixed-2, and Mixed-3, respectively.

The sequencing quality was assessed using FASTQC (Andrew, 2010) and the reads were quality trimmed using Trimmomatic (Bolger et al., 2014; for low Q-scores, adapter contamination and systematic sequencing errors). After quality trimming, only the fragments that retained the forward and reverse reads were kept, any reads with a length less than $36 \mathrm{bp}$ after quality trimming were discarded. For all three samples, the quality trimming step resulted in a $\sim 3 \%$ data loss.

The reads were then aligned to the Mus Musculus GRCm38.p4 reference genome assembly using TopHat (Kim et al., 2013) version 2.1.0. Overall alignment rates were $97 \%$ for Mixed-1, 98\% for Mixed-2, and $97 \%$ for Mixed-3. The resulting BAM alignment files were then passed through Cufflinks (Trapnell et al., 2012) version 2.2.1 in order to calculate gene level FPKM values.

\section{Read Alignment and Methylation Calls}

Enhanced reduced representation bisulfite sequencing reads were mapped on the mouse genome ( $\mathrm{mm} 10)$ with the use of the BSMAP mapping tool (Xi and Li, 2009). The percentages of DNA methylation levels based on bisulfite conversation yield were computed at the single-nucleotide scale. We simulated a range of genome coverage from 5 to 20 and applied statistical analysis of differential methylation which demonstrated that only positions represented by at least $10 \times$ coverage (i.e., $\mathrm{CpG}_{10}$ ) yielded a dataset that had sufficient statistical power for differential methylation calls and allowed for a maximal number of CpG sites to be analyzed, similar to analysis carried out by others (Akalin et al., 2012). We were able to establish the DNA methylation state for 7,492,706 Cytosine positions, including 1,519,053 Cytosine positions from $\mathrm{CpG}$ dinucleotides at $\mathrm{CpG}_{10}$ across all four mouse liver samples. DNA methylation levels of the different genomic elements were computed as a mean of percentages of DNA methylation levels for all $\mathrm{CpG}$ dinucleotides, for which data were available in these regions.

\section{Methylation Comparison and Statistical Analysis}

Percent methylation values for $\mathrm{CpG}$ dinucleotides were calculated by dividing the number of methylated Cs by sequencing depth on that base. For representation as a histogram, percentages were grouped within a 10-percentile range from 0 to $100 \%$ methylation. The methylation score for each of CpG dinucleotides that were covered by at least 10 reads in all samples (Mixed-1, Mixed-2, B6-1, and B6-2) was determined and then we used Pearson correlation distance and Ward's agglomeration method to determine how similar methylation levels were for each $\mathrm{CpG}$ across these samples. Hierarchical clustering of the four samples was performed using the hclust function in R-3.2.1 $1^{1}$. Locally weighted polynomial regression (Lowess) was performed in methylation status scatterplots between each two samples to check their relationships. RRBS datasets for zebrafish brain (GSE59916), liver (GSM1456413; Chatterjee et al., 2014), and mouse brain (GSM1069659) were obtained from Gene Expression Omnibus (GEO). Fisher's exact test was adopted for testing the methylation pattern difference between mouse and zebrafish. The mouse in the brain study is the C57BL/6 strain, brain tissue was collected at 11 weeks. The zebrafish liver methylome was a pool of five male and five female liver harvested from adult fish and the zebrafish brain methylome was from a pool of two males and two females (Chatterjee et al., 2014).

To annotate the genomic feature associate with each $\mathrm{CpG}_{10}$ site, both gene information (promoter, exon, intron, and intergenic) and the list of $\mathrm{CpG}$ islands were retrieved from UCSC table browser ${ }^{2}$. Specific loci were visualized using the epigenome browser $^{3}$ (Zhou et al., 2014). CpG shores were defined as 2,000 bp flanking regions on both upstream and downstream of given $\mathrm{CpG}$ islands (Irizarry et al., 2009). Regions $>2 \mathrm{~kb}$ away from CpG islands are defined as "open sea." If a $\mathrm{CpG}$ shore overlapped with another island, the shores were trucked. If multiple shores were overlapping, they were merged into a single shore. Based on refseq annotation, $\mathrm{CpG}$ dinucleotides were classified into promoter, intron, exon, and intergenic regions. The statistical test

\footnotetext{
${ }^{1}$ http://www.r-project.org/

${ }^{2}$ https://genome.ucsc.edu/

${ }^{3} \mathrm{http}: / /$ epigenomegateway.wustl.edu/browser/
} 
used for the distribution of different genetic elements was Fisher's exact test. TSS regions were defined as the 2,000 bp window centered on the transcript start site (TSS) of genes. In practice, the length of TSS regions is not equal. For global viewing, we calculated the average methylation status ( $\mathrm{mCpG} / \mathrm{CpG}$ ) and $\mathrm{CpG}$ density within each subset of TSS regions and divided into 40 bins equally in the TSS plot.

\section{Integration of DNA Methylation and Gene Expression}

Each $\mathrm{CpG}_{10}$ site was categorized by its methylation status as hypomethylated $(<20 \%$ of reads are methylated $)$ or hypermethylated $(>80 \%$ of reads were methylated). We displayed the relative expression of genes with methylated and unmethylated $\mathrm{CpGs}$ overlapping with promoter regions and gene bodies in according to two common definitions: $5 \mathrm{~kb}$ upstream of the TSS and the $\pm 2 \mathrm{~kb}$ of the TSS which includes CpGs in gene bodies. Within each region, all methylated and unmethylated CpG were counted to generate methylated and unmethylated gene lists. Then, genes were ranked based on the number of CpG sites. Gene expression was represented by FPKM values and FPKM values were log transformed to display in the heat map.

\section{RESULTS}

\section{Mouse Liver Methylome Mapped by ERRBS}

Many studies in mice and humans have described widespread methylome differences in the same organ under different physiological and pathological states (Bird, 2002; Spiers et al., 2015; Zhang et al., 2015). To determine whether differences between background strains or housing conditions altered the hepatic methylome, we compared ERRBS datasets from two male mice on a mixed background (mixed-1, mixed-2) and two males on a pure Black-6 (B6-1, B62) background. Mice from different strains were housed in vivariums at different institutions. As expected, ERRBS enriched for CpG sites in the mouse liver methylome (Table 1). ERRBS does not distinguish between $5 \mathrm{mC}$ and $5 \mathrm{hmC}$, and thus we are unable to differentiate the potential differential contribution of these two modifications. However, given that previous studies found a very low level of $5 \mathrm{hmC}$ in liver $(0.03-0.06 \%$ of $\mathrm{dG})$ compared to the central nervous system and the spinal cord $(0.3-0.7 \%)$ in mouse (Globisch et al., 2010), we reasoned that $5 \mathrm{hmC}$ only contributed a small proportion of all of our methylated C calls.

A previous study simulated the size of reduced representation genome in the mouse as 1.5 million $\mathrm{CpG}$ sites, which is around 1.4 percent of whole genome; this represents 7.0 percent of total genomic CpG sites (Chatterjee et al., 2013). Here, CpG sites covered were calculated as (total CpGs detected)/(average CpG coverage) and thus the number of $\mathrm{CpG}$ sites ranged from 76.1 to 104.7 million reads which represents, on average, $10 \%$ of total genomic CpG sites (Table 1). Of these, the number of CpG sites covered by at least 10 reads $\left(\mathrm{CpG}_{10}\right)$ ranged from 1.33 to 1.58 million (Table 1). The mean $\mathrm{CpG}$ coverage depth ranged from 44 to 64 across the four samples. Non-CpG cytosines $(\mathrm{CpH})$ were rarely methylated $(0.2 \%$ compared with $28 \%$ methylation for CpG cytosines, Table 1). Since our data confirmed that ERRBS enriches for CpGs, non-CpG methylation was not considered further. Thus, our dataset represents a sample of roughly $10 \%$ of the CpGs in the genome, albeit non-randomly distributed across the genome. Nevertheless, we consider this as a representative sample for comparative methylome analysis and provide a resource for investigation into changes in DNA methylation patterns in liver disease, tumorigenesis, and regeneration in mice.

\section{DNA Methylation Pattern Is Consistent across Mouse Strains}

We found that total $\mathrm{CpG}$ methylation is strikingly similar among the four mouse liver samples from two different genetic backgrounds, ranging from $26.6 \%$ to $28.7 \%$ ( $p>0.05$ between every two strains; Table 1). Analysis of all $\mathrm{CpG}_{10}$ sites showed a bimodal distribution of methylation, with nearly $85 \%$ of $\mathrm{CpG}_{10}$ categorized as either hyper-methylated, defined as $>80 \%$ of CpGs methylated, or hypo-methylated, defined as $<20 \%$ methylated. We found remarkable consistency in the methylation patterns, with all samples having $25 \%$ of $\mathrm{CpG}_{10}$ defined as hypermethylated and $60 \%$ as hypomethylated (Figure 1). Between 10.4 and $11.4 \%$ of the $\mathrm{CpG}_{10}$ showed intermediate methylation ( $>20 \%$ and $<80 \%$ methylated; Figure 1).

Reduced representation bisulfite sequencing provides $\mathrm{CpG}$ rich regions. In mammalian genomes, it has been shown that $\mathrm{CpGis}$ are enriched in annotated gene promoters. Since these CpGis largely unmethylated (Meissner et al., 2008; Illingworth and Bird, 2009), it is expected that the percent methylation found in an RRBS dataset is lower than the average methylation of the whole genome. A methylome study on rat dorsal root ganglia with RRBS, which analyzed 2.8 million CpG sites, demonstrated hypomethylation (0-10\% methylated) at more than half of the CpG sites and hypermethylation (90-100\% methylated) at about $20 \%$ of sites (Hartung et al., 2012). This pattern is similar to what we found in mouse liver. RRBS in mouse embryonic stem cells (mESC) covered 543,678 $\mathrm{CpG}_{10}$. These results also displayed a similar methylation pattern to our data in that $>40 \%$ of $\mathrm{CpG}$ sites displayed hypomethylation (Meissner et al., 2008). Therefore, the mouse liver methylome analyzed by ERRBS in our study is similar to RRBS performed on other mouse samples.

A global chromosome scatter plot of $\mathrm{CpG}_{10}$ sites revealed a high correlation between methylation levels at each $\mathrm{CpG}$ analyzed across different mouse livers, with Pearson's correlation coefficient between 0.98 and 0.99 (Figure 2A). Subsequently, we generated scatter plots comparing all detected $\mathrm{CpG}$ sites on each chromosome between any two samples (Figure $\mathbf{2 B}$ and Supplementary Figure S1). In this analysis, Mixed-1 and B6-1, which represent two samples from different genetic backgrounds, had the lowest Pearson's coefficient (Figures 2A,B). Scatter plots of paired CpGs from these two samples were displayed by chromosome (Figure 2B), demonstrating their relationships were highly positively correlated, which is, in part, attributed to 
TABLE 1 | The liver methylome is highly consistent across mouse strains.

\begin{tabular}{|c|c|c|c|c|}
\hline & Mixed_1 & Mixed_2 & B6_1 & B6_2 \\
\hline Number of reads & $36,624,797$ & $32,664,237$ & $50,483,884$ & $49,082,208$ \\
\hline Mapping efficiency & $68.3 \%$ & $67.4 \%$ & $67.6 \%$ & $67.7 \%$ \\
\hline Conversion rate & $99.86 \%$ & $99.89 \%$ & $99.88 \%$ & $99.89 \%$ \\
\hline Total \# of Cytosine sequenced & $336,286,562$ & $303,362,122$ & $473,186,484$ & $450,385,315$ \\
\hline Total \# of CpG sequenced & $76,066,210$ & $70,835,055$ & $104,716,169$ & $98,093,922$ \\
\hline \# of CpGs covered >10x & $1,372,559(1.80 \%)$ & $1,329,761(1.88 \%)$ & $1,557,387$ (1.49\%) & $1,528,130(1.56 \%)$ \\
\hline Mean CpG coverage depth $(10 \times)$ & 44 & 49 & 64 & 60 \\
\hline CpG/total C & $22.62 \%$ & $23.35 \%$ & $22.13 \%$ & $21.78 \%$ \\
\hline CpG methylation (mCpG/CpG) & $28 \%$ & $26.6 \%$ & $27.4 \%$ & $28.7 \%$ \\
\hline Non-CpG methylation & $0.3 \%$ & $0.2 \%$ & $0.2 \%$ & $0.2 \%$ \\
\hline
\end{tabular}

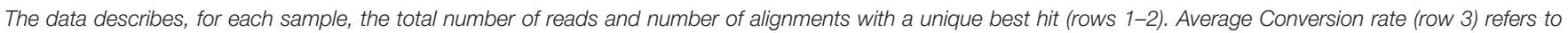

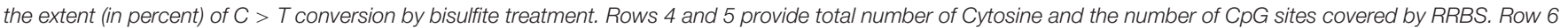

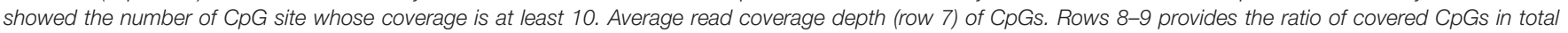

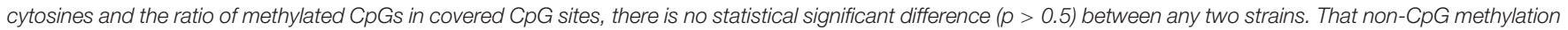
is underrepresented in RRBS is indicated in row 10.

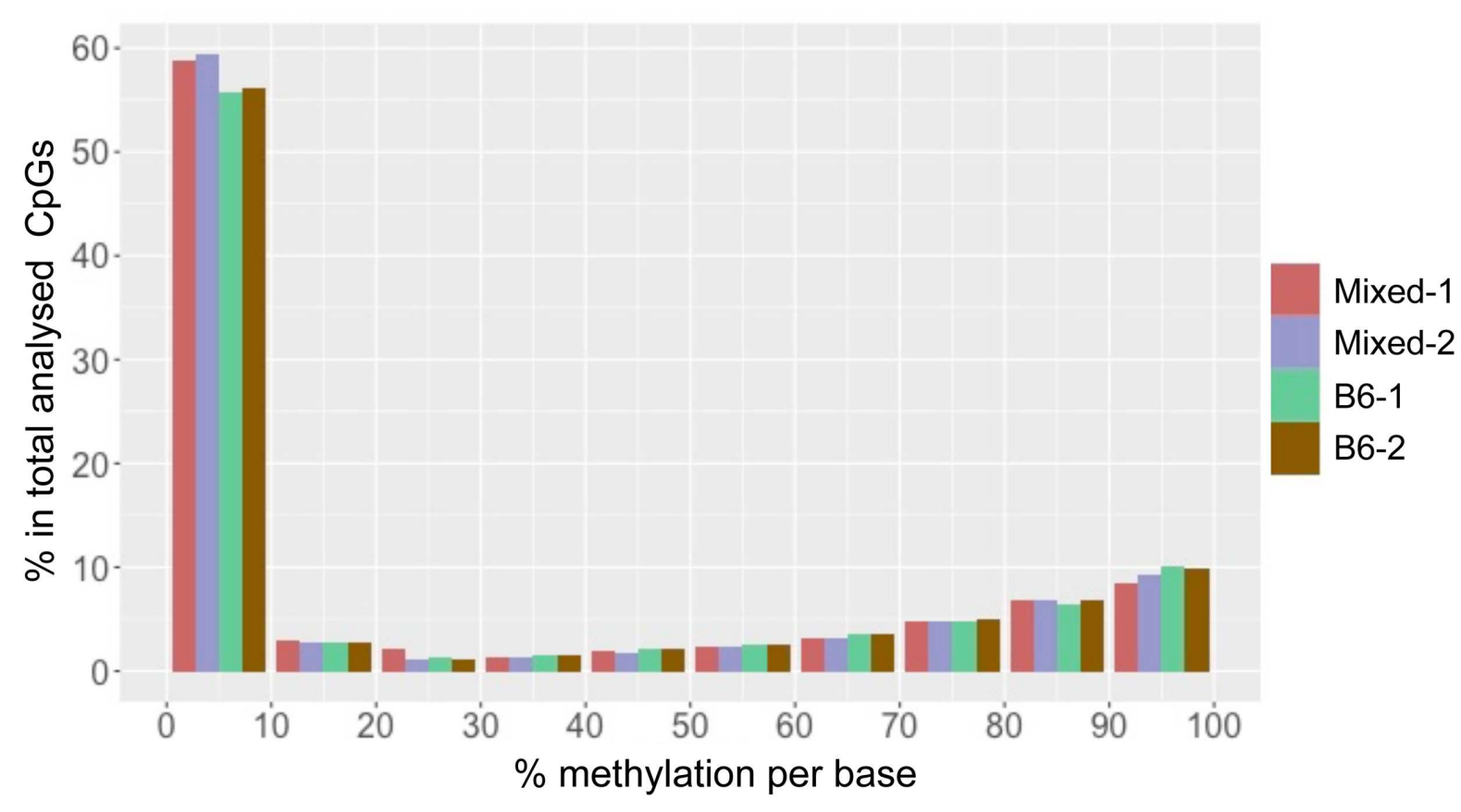

FIGURE 1 | CpG methylation patterns in mouse liver is consistent across different strains. The $x$-axis shows percent methylation for each CpG site. The $y$-axis denotes the percentage of CpGs contained in the corresponding bins. Two male mice from either a Mixed or C57BL/6J (B6) are depicted as with red, purple, green and brown, respectively.

the high depth of coverage of these samples (Table 1). On the other hand, considering variance brought by different strains, we also did further analysis of the regions that are variable between different strains and found that some CpGs methylation are indeed more correlated within strain than inter strains (Supplementary Figure S1). But this kind of variable CpGs only account less than $0.05 \%$ of total CpG10, which is too rare to change the global pattern.

Scatter plots with this pattern were also formed between comparisons of all the other samples (Supplementary Figures S2A,B). This data demonstrates a remarkable similarity in the methylation profile of male mice, regardless of strain and housing differences.

\section{The Intra Species Cpg Methylation Pattern Is Highly Conserved}

In general, comparative transcriptome studies have found that gene expression patterns are similar in the same organs across different species and embryos at comparable developmental stages have common gene expression profiles which are, in part, thought to be mediated by methylation patterns in enhancers (Bogdanović et al., 2016), whereas 
A

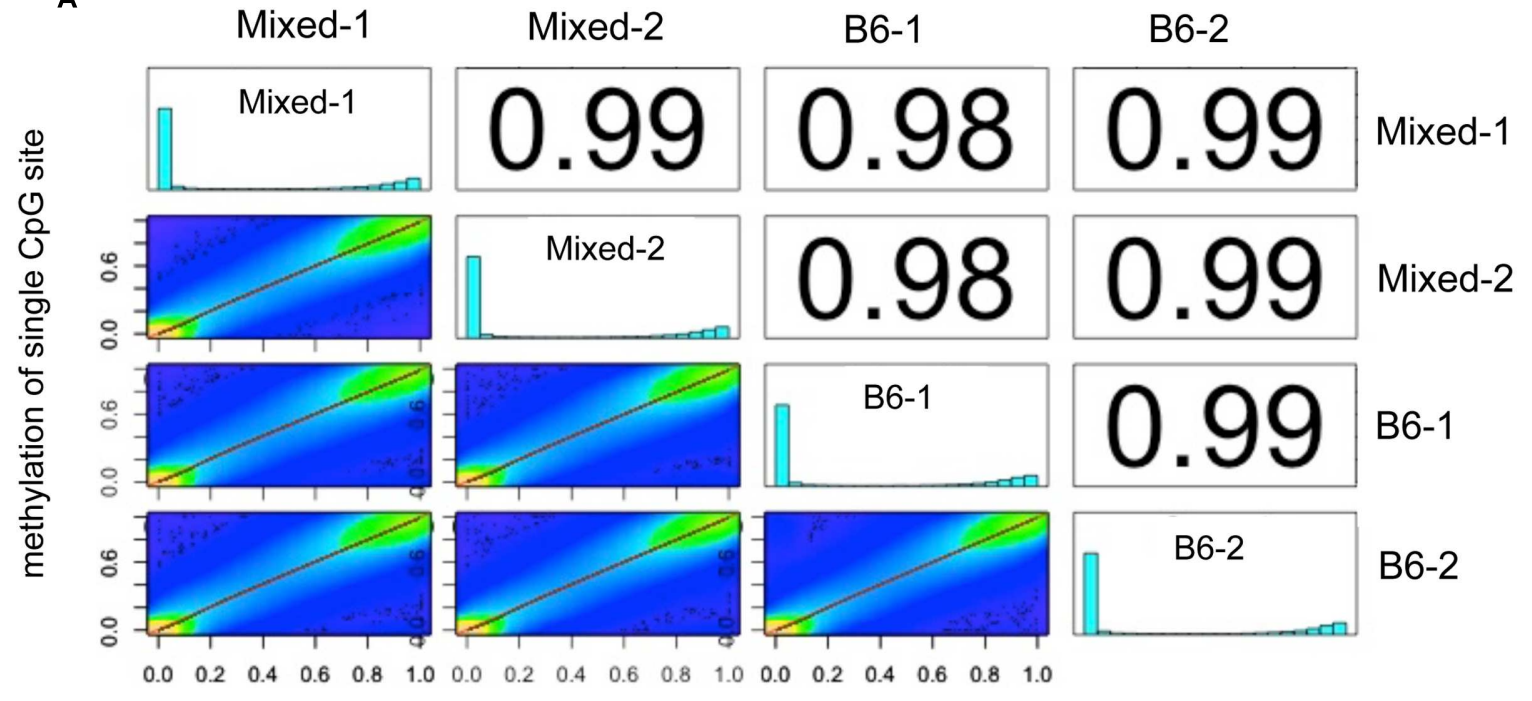

B

methylation of single CpG site

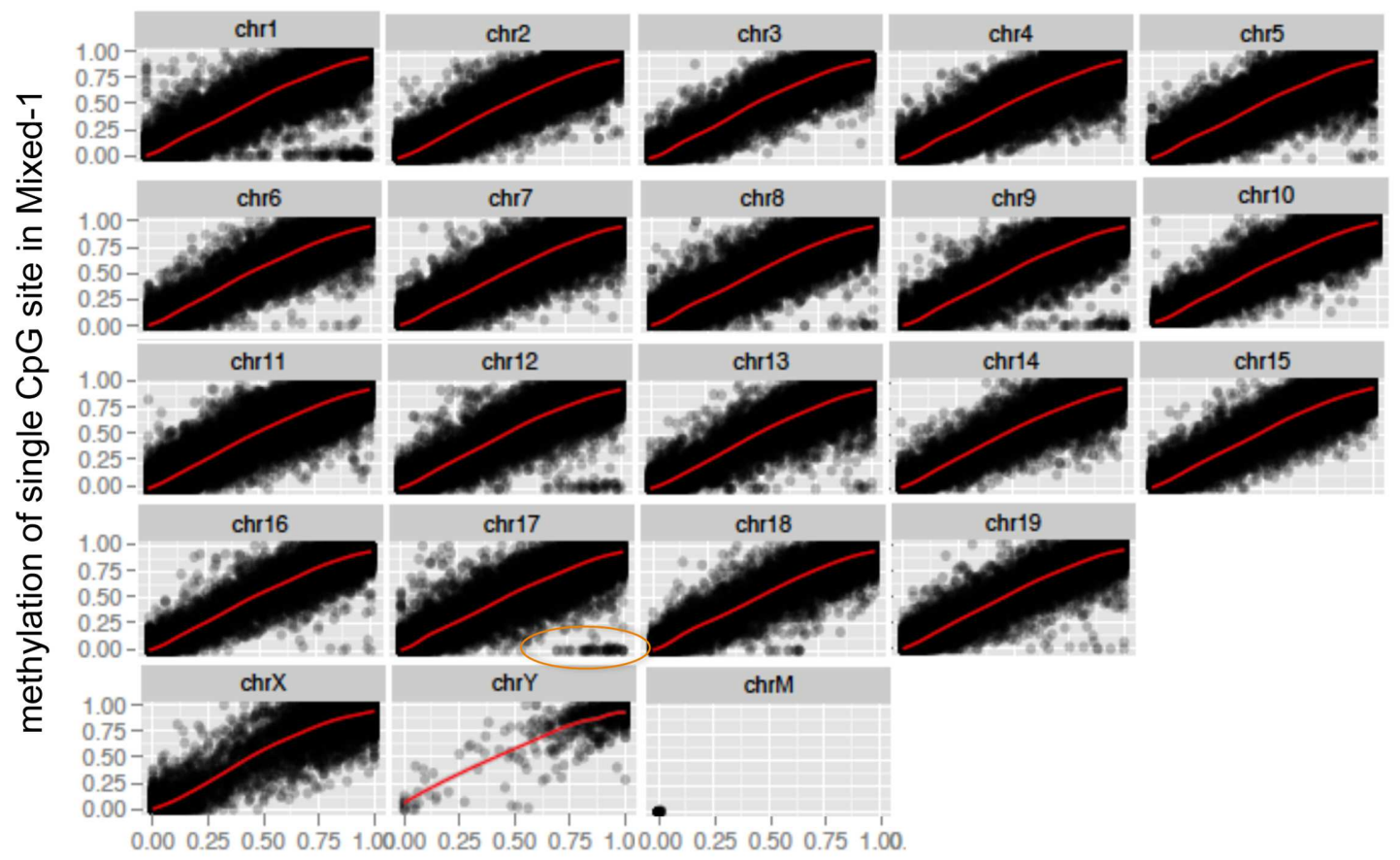

methylation of single $\mathrm{CpG}$ site in B6-1

FIGURE 2 | Global chromosome scatter plots of $\mathbf{C p G}_{10}$ sites in mouse. (A) Scatter plot and correlation of $\mathrm{CpG}$ methylation across four mice. Numbers on the upper right corner denote pair-wise Pearson's correlation scores. Histograms on the diagonal denote distribution of methylation patterns of CpG sites for each stain. (B) Scatter plots of paired CpGs by chromosome, which was aligned between Mixed-1 and B6-1. Differentiated CpGs in orange ellipse go for further analysis in Supplementary Figure S1.

there are dramatic differences in gene expression between different organs from the same species (Zheng-Bradley et al., 2010; Gu et al., 2016). To test whether the same patterns hold true for global $\mathrm{CpG}$ methylation, we retrieved and analyzed RRBS data sets from GEO profiling methylomes of zebrafish liver (GSM1456413) the brain from mouse (GSM1069659) and zebrafish (GSE59916; Chatterjee et al., 2014).

As in mouse liver and in many other species, the methylome in zebrafish liver displayed a bimodal methylation distribution, 
with over $60 \%$ of CpGs categorized as methylated $(>80 \%$ methylation) and $15 \%$ of CpGs as unmethylated $(<20 \%$ methylation), however, with an opposite pattern: the zebrafish liver showed much higher prevalence of hypermethylated $\mathrm{CpG}$ than mouse liver $(p<0.01$; Table 2$)$, with over $50 \%$ and less than $20 \%$ of hyper and hypo-methylated CpG sites, respectively (Figure $\mathbf{3 A}$, Table 2 ). This is consistent with the finding of between 70 and $85 \%$ methylation in whole zebrafish embryos (Potok et al., 2013; Bogdanović et al., 2016) and over 70\% methylation in adult muscle (Potok et al., 2013).

TABLE 2 | Methylation levels are more consistent across species than across organs.

\begin{tabular}{lccccc}
\hline & \multicolumn{3}{c}{ Hypomethylated CpG (<20\%) } & & \multicolumn{2}{c}{ Hypermethylated CpG $>\mathbf{8 0} \%$} \\
\cline { 2 - 5 } Organ & Liver & Brain & p-value & Liver & Brain \\
\hline Mouse & $62.7 \%$ & $61.1 \%$ & 0.5 & $24.35 \%$ & $24.2 \%$ \\
Zebrafish & $24.1 \%$ & $14.78 \%$ & 0.03 & $63.4 \%$ & $68.9 \%$ \\
$p$-value & $2.35 e-09$ & $2.32 e-14$ & & $2.35 e-09$ & 0.5805 \\
\hline
\end{tabular}

Ratios are the percent of methylated $C p G s$ out of all the $C p G_{10}$ detected. The $p$-value on the right is between liver and brain in mouse and zebrafish. The $p$-value on the bottom is between mouse and zebrafish in liver and brain.

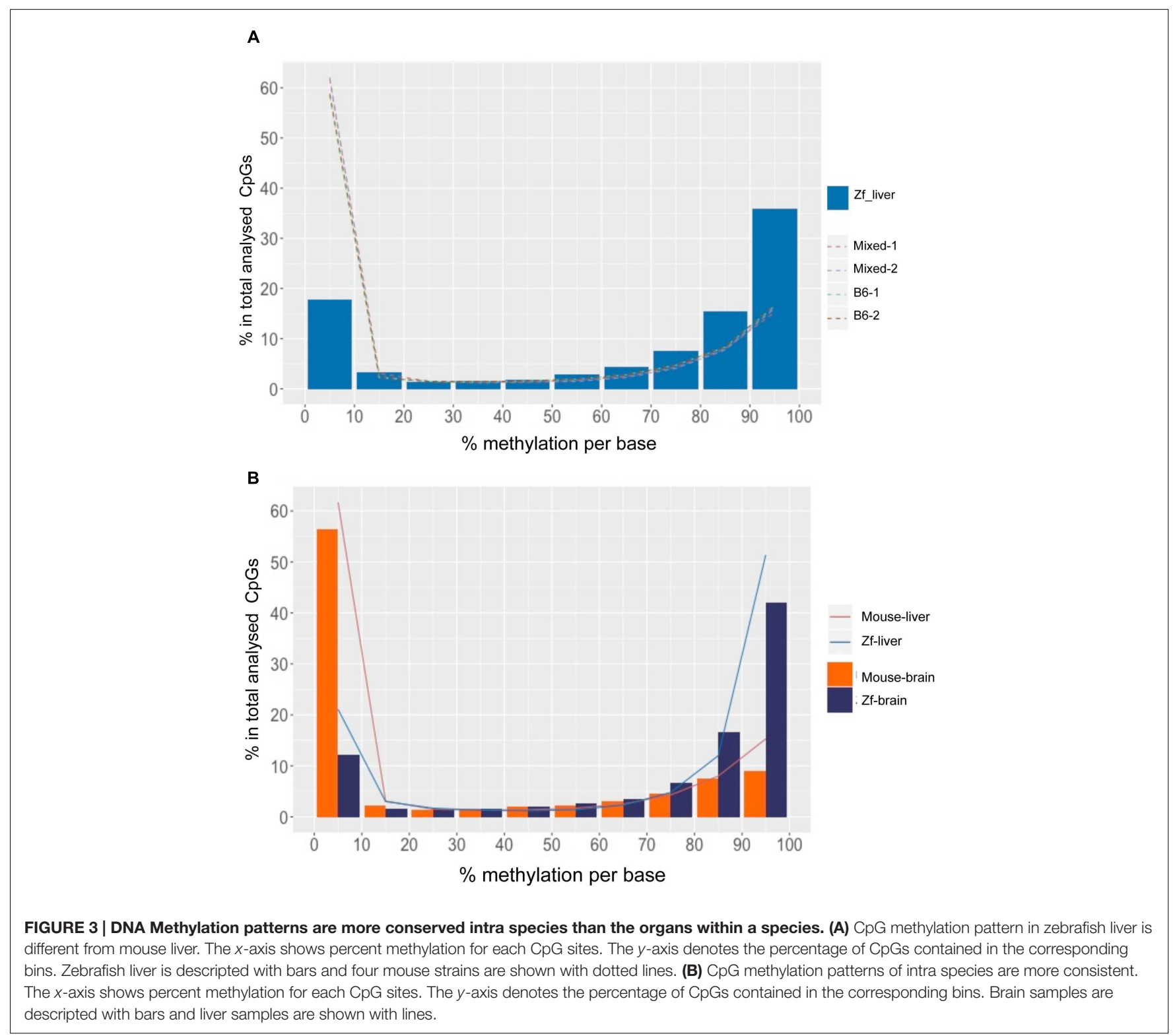


Thus, the zebrafish liver is more highly methylated than the mouse.

To determine whether high levels of methylation in zebrafish could be related to physiological differences between these species, we compared the liver methylomes of both species to that of a very different organ (the brain) within the same species. We found that the same pattern uncovered in the liver was conserved in the brain of both species, with a significantly increased percent of hypermethylated CpGs in zebrafish tissues compared to mouse $(p<0.01$, Figure 3B, Table 2). Intraspecies comparison between brain and liver revealed that the mouse brain displayed similar distribution of methylation levels as the mouse liver $(p>0.05)$, with $61.1 \%$ of $\mathrm{CpG}_{10}$ are hypo-methylated and $24.2 \%$ of hypermethylated (Table 2). This same intraspecies conservation was observed in zebrafish, where over $63.4 \%$ of the $\mathrm{CpG}_{10}$ sites were hyper-methylated and less that $24.1 \%$ were hypomethylated in both organs (Table 2). Although slightly higher levels of hyper-methylated $\mathrm{CpGs}$ were observed in the zebrafish brain $(p>0.01)$, global CpG methylation distributions are still highly consistent between liver and brain of zebrafish (Figure 3A). Based on methylome comparisons between different species, different genetic backgrounds, and different organs, we concluded that CpG methylation patterns are more conserved between different organs within a species than between different species for the same organ. Moreover, consistent with findings from other species (Feng et al., 2010; Bogdanović et al., 2016), in both mouse and zebrafish, CpG methylation conforms to a bimodal patterns whereby cytosines are either entirely methylated or unmethylated.

\section{Hepatic Methylome Is Enriched in Intragenic Regions and Introns}

To determine if the landscape of methylated CpGs differed between mouse and zebrafish, we compared their distribution relative to the genomic features of hepatic methylomes in both species. All analyzed $\mathrm{CpG}_{10}$ sites in both liver datasets were classified into annotated regions. In the mouse, $52 \%$ of the $\mathrm{CpG}_{10}$ dinucleotides were in promoter regions and 55\% were in CpGi (Figures $\mathbf{4 A}, \mathbf{C}$ ). This is consistent with the observation that most $\mathrm{CpGis}$ are found near sites of transcription initiation (Deaton and Bird, 2011). Other CpG sites are found in exons, introns, and intergenic regions accounting for 10, 16, and $22 \%$ of total CpGs levels, respectively (Figure $4 \mathbf{A}$ ). We categorized hypermethylated CpGs as those with $>80 \%$ of the reads as methylated and hypomethylated CpGs as $<20 \%$ of reads as unmethylated. Hypermethylated $\mathrm{CpGs}$ were intergenic and intronic $\mathrm{CpG}_{10}$ (43 and 35\%, respectively; $p<0.01$; Figure 4A), and excluded from promoters, and $78 \%$ of hypo-methylated $\mathrm{CpG}_{10}$ were in annotated promoters (Figure 4A). This, in part, is reflective of the distribution of the $\mathrm{CpG}_{10}$ in the mouse

\section{mouse}

A

\section{mouse}

C

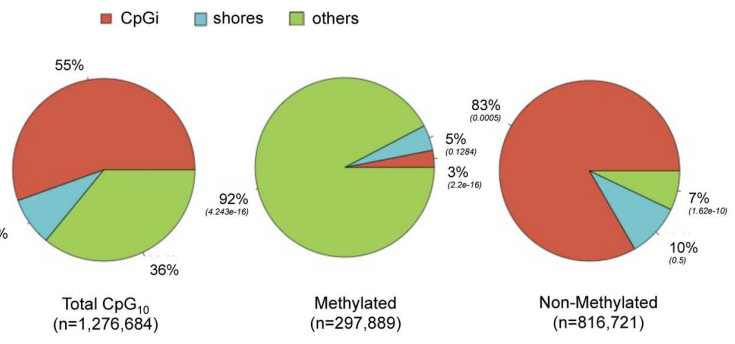

zebrafish

\section{zebrafish}

B

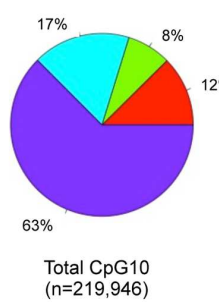

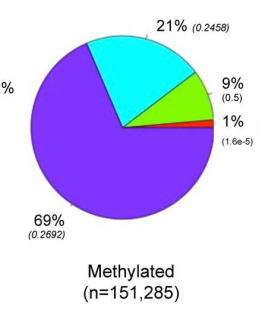

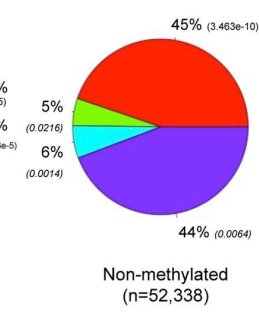

D

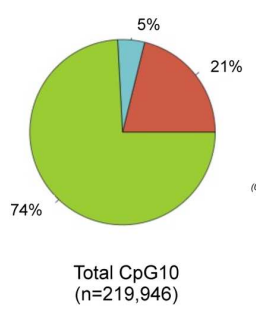

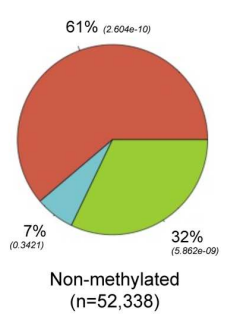

FIGURE 4 | There are marked differences in the methylome landscapes between the zebrafish and mouse hepatic methylome. Total CpG 10 is displayed in the left pie of each panel. Methylated is defined as $>80 \% \mathrm{CpGs}$ methylated and non-methylated is defined as $<20 \%$ methylated. $P$-value (Chi-square test) in parentheses is to total $\mathrm{CpG}_{10}$. (A) Gene body for mouse. (B) Gene body for zebrafish. (C) CpG elements for mouse. (D) CpG elements for zebrafish. 
A

mm10 genome based

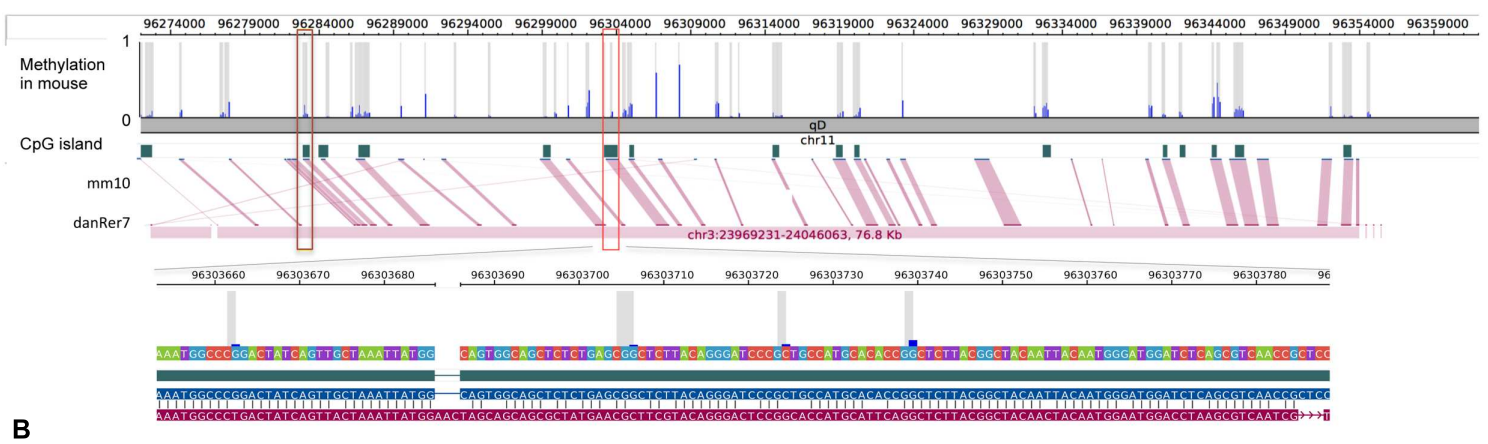

danRer7 genome based

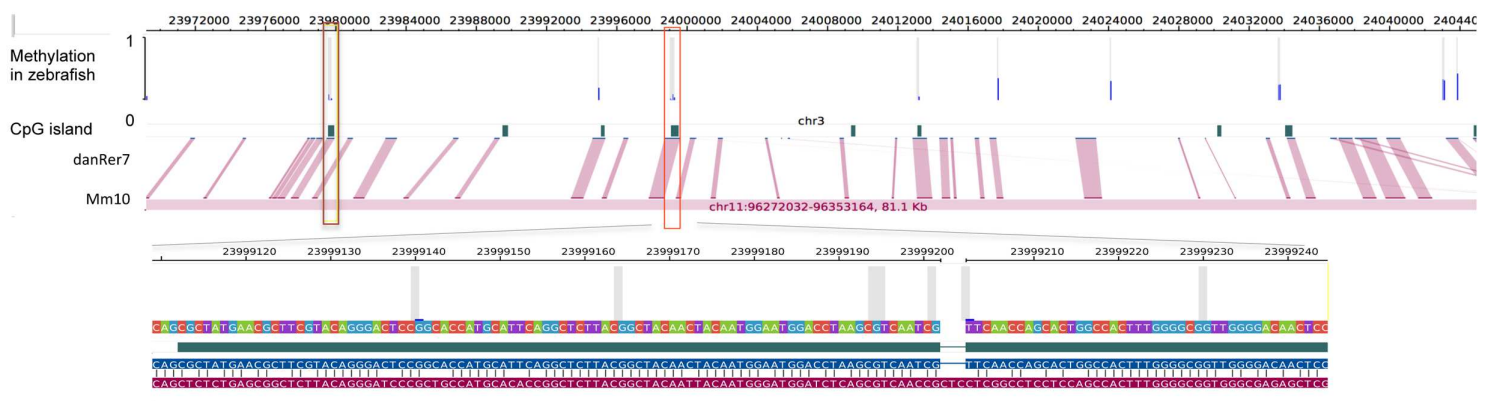

FIGURE 5 | Methylation of shared CpG islands in mouse and zebrafish genome are overall at a low level. Each detected CpG site is marked with gray bar and methylation status is shown by blue bar ranging from 0 to 1. CpG islands are annotated based on reference genome mm10 (A) and danRer7 (B), respectively. Pinked lines link corresponding CpG islands, two orthologous $\mathrm{CpG}$ islands are marked with brown and red squares.

TABLE 3 | There is a higher prevalence of repeats in the zebrafish genome compared to the mouse.

\begin{tabular}{lll}
\hline & Mouse (Mm10) & Zebrafish (GRCz10) \\
\hline Genome size & $2,652,783,500 \mathrm{bp}$ & $1,464,443,456 \mathrm{bp}$ \\
Total bp in TEs & $1,201,953,154 \mathrm{bp}$ & $756,790,665 \mathrm{bp}$ \\
& $(45.3 \%)$ & $(51.8 \%)$ \\
TEs overlap with CpGi & $413,896(0.028 \%)$ & $32,416(0.004 \%)$ \\
TEs overlap with CpG10 & $274,594(17.63 \%)$ & $117,032(53.21 \%)^{*}$
\end{tabular}

TEs are the repeat sequence screened by RepeatMasker, the ratio is out of corresponding genome. In zebrafish TEs is significantly higher prevalent that mouse ( $p=0.0323$ ). CpGi is annotated from UCSC table browser, the ratio in bracket is out of TEs in row 2. CpG10 are those analyzed in our datasets, ration accounts for TEs in row 2. There are significant more TEs overlapping with CpG10 in zebrafish than in mouse (marked with asterisk, $p<0.01$ ).

dataset, of which $52 \%$ were mapped to annotated promoters (Figure 4A).

The zebrafish hepatic methylation landscape was strikingly different, with $63 \%$ of all $\mathrm{CpG}_{10}$ falling within intergenic regions (compared to $22 \%$ in mouse) and only $12 \%$ within promoter regions (compared to $52 \%$ in mouse; Figure $4 \mathrm{~B}$ ). The methylated $\mathrm{CpG}_{10}$ in zebrafish largely mirrors the genomic distribution of $\mathrm{CpGs}$ in this dataset: the majority are found in intragenic regions and introns (69 and 21\%, respectively). Promoter regions are the exception to the pattern, as there is a significant exclusion of methylated CpGs from zebrafish promoters $\left(p=1.6 \times 10^{-6}\right.$; Figure $\left.4 \mathbf{B}\right)$, similar to the pattern in the mouse hepatic methylome (Figure 4A). Other similarities between species include a relative low percent of methylated $\mathrm{CpG}_{10}$ in $\mathrm{CpGis}$ (21\% in zebrafish; 55\% in mouse; Figures 4C,D) and that the vast majority of methylated $\mathrm{CpG}_{10}$ were found in shores and other regions (i.e., open sea), respectively (Figures 4C,D). Therefore, although there are striking differences in the methylome landscape between the same tissue in these two species, the finding that $\mathrm{CpGis}$ are in general, not methylated (i.e., NMIs), is consistent with findings from other species (Irizarry, 2009; Zemach et al., 2010; Long et al., 2013). We examined several orthologous $\mathrm{CpG}$ islands in mouse and zebrafish genome to determine whether the level of methylation was conserved across similar genomic regions. Given that methylation of $\mathrm{CpGis}$ is, in general, very low, (Figures 4C,D), many orthologous CpGs are unmethylated in the liver of both species. One example is the orthologous $\mathrm{CpGi}$ on chromosome 11 in mice and chromosome 7 in zebrafish, which was unmethylated in both species (Figure 5).

We found a much higher percent of CpGs in intragenic regions of the zebrafish genome are methylated in the liver compared to mouse ( $82 \%$ vs. $60 \%$, respectively). This is consistent with the overall higher level of TEs in the zebrafish genome (Table 3) and similar to the findings of others (Feng et al., 2010; Potok et al., 2013). To determine whether the high level of methylation in the zebrafish intragenic region was attributed 

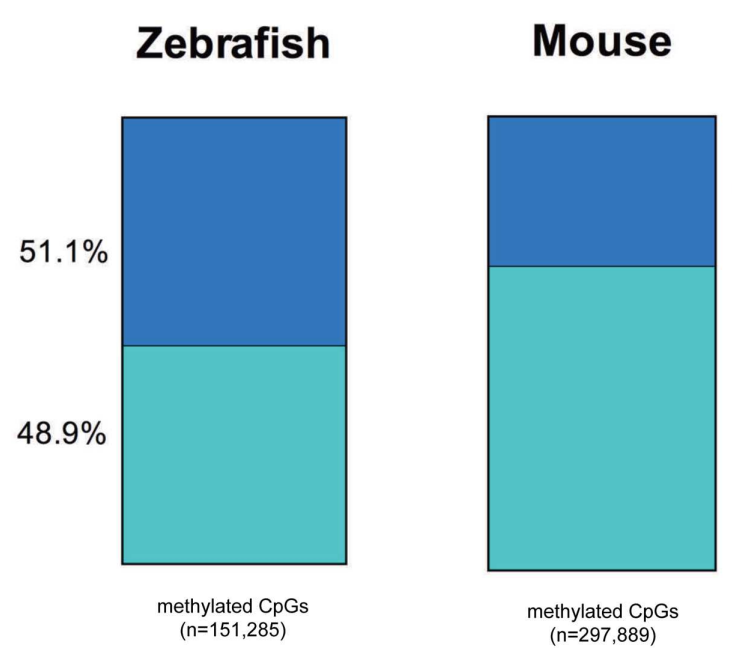

TEs region Non-TEs

$33.01 \%$

$66.99 \%$

FIGURE 6 | More methylated CpGs overlap with TEs in zebrafish liver methylome. All the methylated $\mathrm{CpG}_{10}$ covered are annotated with TEs in mouse and zebrafish genome. TEs-methylated CpGs are displayed with blue and non-TEs methylated CpGs are shown with green. The number of $\mathrm{CpG}$ sites is under the bar.

to TE abundance, we examined where methylated CpGs reside in mouse and zebrafish (Figure 6). We found that more than half of the methylated CpGs in zebrafish are found in TEs, while in the mouse only one third methylated CpGs are in TEs.

It is widely observed that CpGis cluster in promoters and the regions flanking TSS. We found a negative correlation between methylation level and proximity to the TSS in adult liver methylomes in both mouse (Figure 7A) and zebrafish (Figure 7B). Notably, the TSS plot revealed a high correlation between the methylation patterns of the four separate mouse liver samples, confirming our finding that there is little variation in the hepatic methylome within mice.

\section{Correlation between DNA Methylation of Gene Regulatory Regions and Gene Expression}

DNA methylation is proposed to repress gene expression. Given that most promoters are not methylated (Figures 7A,B), it is difficult to envision how methylation could be a fundamental mechanism that regulates gene expression, except in cases that depart from this pattern and have high methylation levels across their promoters. To examine this further, we counted the number of $\mathrm{CpG}_{10}$ within $-5 \mathrm{~kb}$ upstream of the TSS and $\pm 2 \mathrm{~kb}$ TSS regions that were either methylated ( $>80 \%$ of reads methylated) or unmethylated ( $<20 \%$ of reads as methylated), and those with the highest number of methylated $\mathrm{CpG}_{10}$ were counted as "hypermethylated" genes and those with the highest number of unmethylated $\mathrm{CpG}_{10}$ were counted as "hypomethylated" genes (Supplementary Table 1). Genes with no CpGs were excluded. In both gene lists, we found CpGs are enriched in the TSS regions, but methylation status is different. Examples of genes in both categories are shown in Supplementary Figure S3. We noted that there are far fewer CpGs in genes categorized as hypermethylated compared to hypomethylated genes (Supplementary Tables S1 and S2). This indicates that regions with lower CpG content are higher methylated than those with more CpGs, consistent with the finding of others (Lee et al., 2015).

We carried out RNAseq analysis on samples from the Mix-1 and Mix-2 livers used for ERRBS as well as of a third sample from a third male mouse from the same background and queried the expression of genes annotated as high and low methylated. We showed the top 100 genes from the methylated and unmethylated gene lists of $-5 \mathrm{~kb}$ upstream of the TSS in Figure 8. To include CpG methylation information in gene bodies, we also count methylated and unmethylated genes based on $\pm 2 \mathrm{~kb}$ TSS regions (Supplementary Figure S4). Overall, we found in both categories there are more genes in the unmethylated list that are expressed at higher levels than those in the methylated category (Figure 8 and Supplementary Figure S4) However, there are numerous exceptions, as many of the genes categorized as methylated were expressed at high levels, and vise versa, many of the genes which had very low methylation level were expressed also at relative low levels. Together, these data are consistent with findings across a range of species where there is a modest trend in the correlation between promoter methylation and gene expression, but clearly shows that methylation is neither necessary nor sufficient to suppress expression of many genes in the mouse liver.

\section{DISCUSSION}

Reduced representation bisulfite sequencing is a cost effective approach to study global DNA methylation by enriching GCrich genomic regions. Although a significant number of non-CpG loci can also be detected by RRBS, the overlap between RRBS and whole genome bisulfite sequencing is much greater for $\mathrm{CpG}$ loci than non-CpG loci. A study on human embryonic stem cells (hESCs) by whole genome sequencing detected 830,000 CpG sites (Ziller et al., 2011). On the other hand, whole genome bisulfite 


\section{A}

\section{mouse}
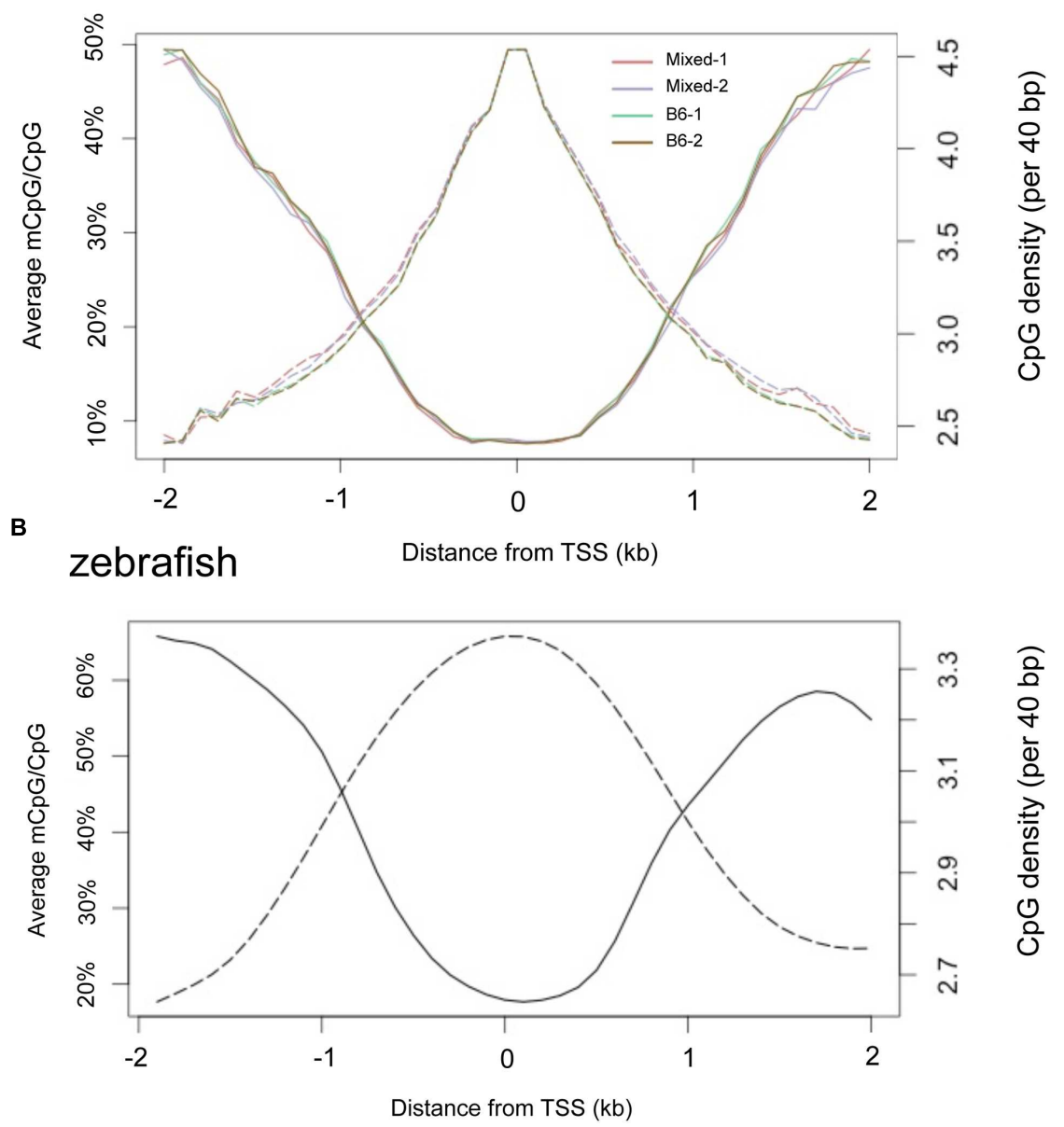

FIGURE 7 | TSS regions have higher CpG density and less methylation in both mouse and zebrafish livers. Composite profiles the regions are $2 \mathrm{~kb}$ around TSS. $Y$-axis on the left denotes the average of methylated $\mathrm{CpG}$, represented by a solid line. $Y$-axis on the right hand denotes the CpG density, represented by dashed lines. (A) Livers from four mice strains. (B) Zebrafish liver.

sequencing across human cell types detected 250,000 non-CpG loci while RRBS detected 213,000 non-CpG loci with an overlap of 52,000 loci (<25\%; Ziller et al., 2011). These observations support the use of RRBS as an excellent method for measuring CpG methylation.

This being said, RRBS has limited coverage of intergenic regions and $\mathrm{CpGi}$ shores, which are important genomic features. ERRBS is enhanced RRBS, which has been used to resolve DNA methylation patterns in human and other animals (GarrettBakelman et al., 2015). It is an optimized RRBS by two ways: (1) biochemically, fragments size selection is optimized to capture more CpG sites by the combination of two enzymes, Taq $\alpha \mathrm{I}$ and MspI, and (2) bioinformatically, alignment algorithm is optimized to increase mapping efficiency (Akalin et al., 2012). When compared to RRBS, ERRBS resulted in a higher number of CpGs represented in the data generated and increased coverage of all genomic regions interrogated (Lee et al., 2014). So examining regions of the genome not covered by this method could potentially yield a new perspective on the comparative methylome. A caveat to all bisulfite based methylome approaches is the end is the inability to discriminate between $5 \mathrm{mC}$ and $5 \mathrm{hmC}$, however, given that $5 \mathrm{hmC}$ represents only a small fraction of all methylated CpGs, this is more of a concern when the direct role of specific CpGs is under investigation. Additional consideration for the ERRBS and RRBS datasets is that the distribution of mapped reads is not entirely random. However, considering the considerable advantage in sequencing depth compared to whole genome bisulfite sequencing, (E)RRBS allows analysis of CpGs methylation in each major genomic element and provides an excellent approach for comparative analysis of the methylome landscape.

The current study is among the first comparisons of the methylome from the same tissue of mouse and zebrafish. A previous study focusing on NMI distribution and function among several vertebrates, including mouse and zebrafish, identified more NMIs that were shared between liver and testis 


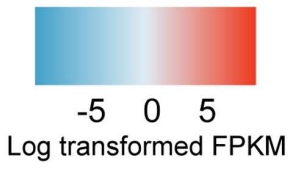

Log transformed FPKM unmethylated
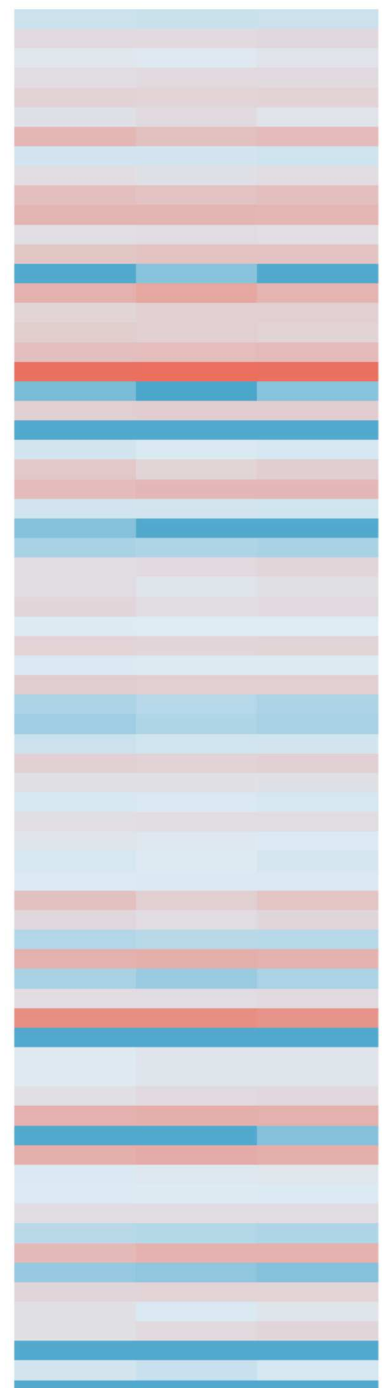

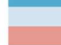

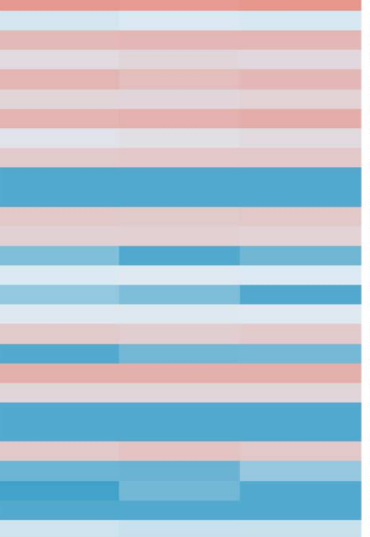

Mix-1 Mix-2 Mix-3

\section{methylated}

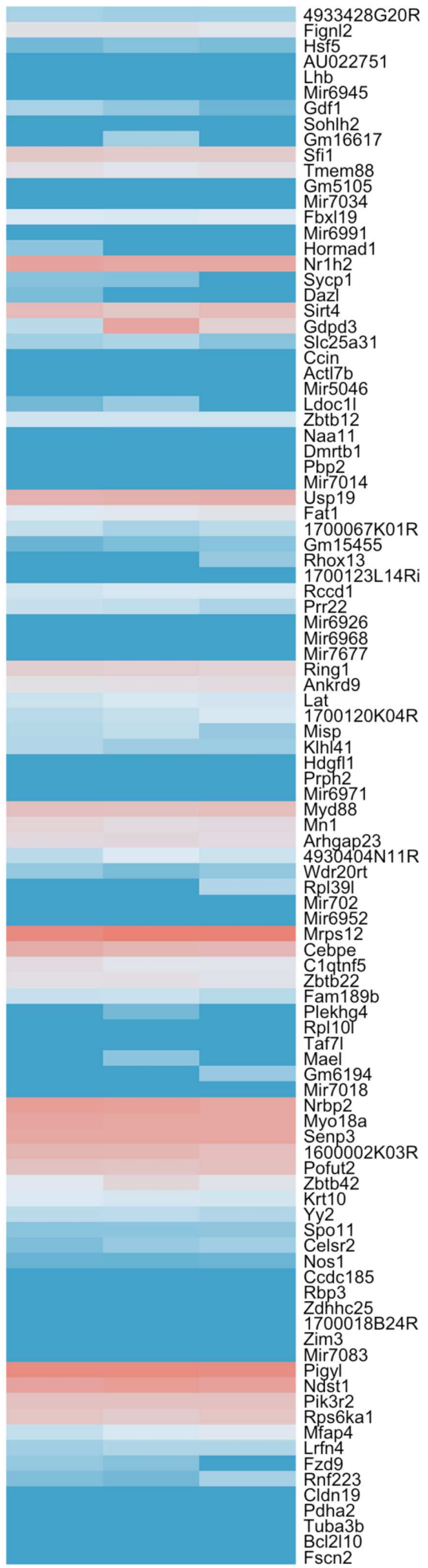

Mix-1 Mix-2 Mix-3

FIGURE 8 | More hypermethylated genes are expressed at lower levels than hypomethylated genes. Top 100 genes ranked in hypermethylated (> $80 \%$ ) and hypomethylated (<20\%) gene list were plotted with expression values. The gene list was based on the counting CpG in promoter regions $(5 \mathrm{~kb}$ upstream of TSS). Color scale was represented by the log transformed fpkm value from RNAseq. 
in zebrafish compared to mouse (18854 vs. 16680, respectively), and an expanded number of unique NMIs in the zebrafish liver compared to those that were unique to zebrafish testis (Long et al., 2013). Interestingly, many genes marked as unique NMIs in testis or liver were differentially expressed in these two tissues in mouse, human chicken and platypus samples (Long et al., 2013). Another study comparing the methylation profile of whole zebrafish and mouse embryos found very high levels of CpG methylation in both (74\% in zebrafish and $80 \%$ in mouse; Feng et al., 2010) and showed a similar pattern of methylated CpGs across gene bodies, repeats and TEs (Feng et al., 2010). The differences between our findings of greatly divergent methylation levels between the mouse and fish hepatic and brain methylomes could be attributed to a difference in tissue studied, as the mouse embryo may have more $\mathrm{CpG}$ methylation than a differentiated tissue, or in the method used to interrogate the methylome. Indeed, a comparison between the methylation patterns in the whole early zebrafish embryo to that of adult differentiated muscle revealed that about $70 \%$ of CpGs were hypermethylated in muscle, a slightly lower level than observed in pre-gastrula embryos at the sphere and 256 cell stage (>90\%; Potok et al., 2013; Bogdanović et al., 2016). Thus, our finding that CpG methylation in zebrafish liver and brain has ranges from 63 to $69 \%$ demonstrates that the zebrafish genome during both embryonic development and in differentiated tissues is highly methylated.

A second observation from our work is that the zebrafish genome has a much higher level of CpG methylation compared to the mouse genome. This is probably attributed to an increased prevalence of intergenic regions in zebrafish than that in mouse, as annotated intergenic regions tend to be enriched for methylated $\mathrm{CpGs}$ in both mouse and zebrafish (see Figures 4 and $\mathbf{6}$ and Table 3). This observation is also in line with the presence of more TEs in intergenic regions, in the zebrafish genome than that in the mouse genome (Kapusta et al., 2013). Considering the genome size and repeat sequence proportion individually, TEs comprise $51.8 \%$ of the zebrafish genome and $45.3 \%$ in the mouse genome (Table 3 ). The finding that the fraction of the genome occupied by TEs is higher in zebrafish can be attributed to the huge amplification of DNA transposons in zebrafish. In fact, the zebrafish is the only vertebrate studied which displays such a dramatic and recent amplification of TEs (Chalopin et al., 2015). It is likely that the zebrafish genome is loaded with potentially active elements, (Lam et al., 1996; Hagemann and Hammer, 2006) which can rapidly amplify their population across the genome and DNA methylation serves as a primary mechanism to silence these. We propose that the high level of DNA methylation in zebrafish is a consequence of this TE-enriched genome and $\mathrm{CpG}$ islands, as a mechanism of limiting transposition. Indeed, we find that most of the methylated CpGs in the intragenic region map to the TEs in zebrafish (Figure 6). In contrast, the mouse genome is dominated by L1 and SINEs, which are also active but most of the progenitors have accumulated inactivating mutations (Kapusta et al., 2013). Thus, it is very likely that the difference in methylation results from the different evolutionary dynamics of TEs in these two genomes (Chalopin et al., 2015).
These findings underscore the concept that the major role for DNA methylation is not only to play prominent role of DNA methylation in regulating gene expression, but also repress expression of repetitive sequences.

\section{AUTHOR CONTRIBUTIONS}

CZ fulfilled major work of this study, including data analysis, making figure and writing manuscript. During this process, KCS and $\mathrm{YH}$ both gave insightful guidance on epigenetic question and bioinformatics methods.

\section{FUNDING}

This work was supported by the NIH (5R01DK080789 to KCS and 1R01DK099558 to YH), Irma T. Hirschl Trust and Dr. Harold and Golden Lamport Research Award supported to $\mathrm{YH}$.

\section{ACKNOWLEDGMENTS}

We are grateful to Chinwe Ukomadu for mouse liver samples and insightful discussions, to Amaia Lumbajio for ERRBS datasets from two male mice, Shuang Wang and Shikha Nayar for careful reading of the manuscript and members of the Sadler and Hoshida labs for helpful discussion. Nizar Drou in the Bioinformatics Core Facility at NYU Abu Dhabi contributed to RNA-seq data. Alicia Alonso in the Epigenomics Core Facility at Weill Cornell Medical College provided valuable suggestions and guidance with obtaining ERRBS data.

\section{SUPPLEMENTARY MATERIAL}

The Supplementary Material for this article can be found online at: http://journal.frontiersin.org/article/10.3389/fgene.2016. 00110

FIGURE S1 | Differentially methylated CpGs between mouse strains are consistent within strains. Scatter plots of differentiated CpGs, which are marked in Figure 2, between Mixed-1 and B6-1 (A), B6-1 and B6-2 (B) and Mixed-1 and Mixed-2 (C).

FIGURE S2 | Global chromosome scatter plots of $\mathrm{CpG}_{10}$ sites in mouse livers from different strain combinations. (A) Scatter plots of paired CpGs by chromosome, which was aligned between Mixed-2 and B6-2. (B) Scatter plots of paired CpGs by chromosome, which was aligned between B6-1 and B6-2.

FIGURE S3 | Representative tss region of Hsf5 and Zswim6 displayed different methylation status. Red bars in the top methylation track showed the status of methylation, whose range is from 0 to 1 . The lower reads coverage track displayed TSS regions are enriched and CpG sites were captured by this study were highlighted by red or green. (A) Hsf5 represents hyper-methylated genes. (B) Zswim6 represents hypo-methylated genes.

FIGURE S4 | Top 100 genes ranked in hypermethylated (> 80\%) and hypomethylated $(<\mathbf{2 0} \%)$ gene list were plotted with expression values. The gene list was based on the counting $\mathrm{CpG}$ sites in promoter regions and gene bodies ( $\pm 2 \mathrm{~kb}$ of TSS). Color scale was represented by the log transformed fpkm value from RNAseq. 


\section{REFERENCES}

Akalin, A., Garrett-Bakelman, F. E., Kormaksson, M., Busuttil, J., Zhang, L., Khrebtukova, I., et al. (2012). Base-pair resolution DNA methylation sequencing reveals profoundly divergent epigenetic landscapes in acute myeloid leukemia. PLoS Genet. 8:e1002781. doi: 10.1371/journal.pgen.1002781

Andrew, S. (2010). FastQC: A Quality Control Tool for High Throughput Sequence Data. Available at: http://www.bioinformatics.babraham.ac.uk/projects/fastqc

Bestor, T. H., Edwards, J. R., and Boulard, M. (2015). Reply to Wilkinson: minor role of programmed methylation and demethylation in mammalian development. Proc. Natl. Acad. Sci. U.S.A. 112, E2117. doi: $10.1073 /$ pnas. 1504199112

Bird, A. (2002). DNA methylation patterns and epigenetic memory. Genes Dev. 16, 6-21. doi: 10.1101/gad.947102

Bogdanović, O., Smits, A. H., de la Calle Mustienes, E., Tena, J. J., Ford, E., Williams, R., et al. (2016). Active DNA demethylation at enhancers during the vertebrate phylotypic period. Nat. Genet. 48, 417-426. doi: 10.1038/ng.3522

Bolger, A. M., Lohse, M., and Usadel, B. (2014). Trimmomatic: a flexible trimmer for Illumina sequence data. Bioinformatics 30, 2114-2120. doi: 10.1093/bioinformatics/btu170

Boumil, R. M., and Lee, J. T. (2001). Forty years of decoding the silence in X-chromosome inactivation. Hum. Mol. Genet. 10, 2225-2232. doi: 10.1093/hmg/10.20.2225

Chalopin, D., Naville, M., Plard, F., Galiana, D., and Volff, J.-N. (2015). Comparative analysis of transposable elements highlights mobilome diversity and evolution in vertebrates. Genome Biol. Evol. 7, 567-580. doi: 10.1093/gbe/evv005

Chatterjee, A., Ozaki, Y., Stockwell, P. A., Horsfield, J. A., Morison, I. M., and Nakagawa, S. (2013). Mapping the zebrafish brain methylome using reduced representation bisulfite sequencing. Epigenetics 8, 979-989. doi: 10.4161/epi.25797

Chatterjee, A., Stockwell, P. A., Horsfield, J. A., Morison, I. M., and Nakagawa, S. (2014). Base-resolution DNA methylation landscape of zebrafish brain and liver. Genomics Data 2, 342-344. doi: 10.1016/j.gdata.2014.10.008

Deaton, A., and Bird, A. (2011). CpG islands and the regulation of transcription. Genes Dev. 25, 1010-1022. doi: 10.1101/gad.2037511.1010

Eden, A., Gaudet, F., Waghmare, A., and Jaenisch, R. (2003). Chromosomal instability and tumors promoted by DNA hypomethylation. Science 300, 455. doi: 10.1126/science.1083557

Elliott, G., Hong, C., Xing, X., Zhou, X., Li, D., Coarfa, C., et al. (2015). Intermediate DNA methylation is a conserved signature of genome regulation. Nat. Commun. 6, 6363. doi: 10.1038/ncomms7363

Feng, S., Cokus, S. J., Zhang, X., Chen, P.-Y., Bostick, M., Goll, M. G., et al. (2010). Conservation and divergence of methylation patterning in plants and animals. Proc. Natl. Acad. Sci. U.S.A. 107, 8689-8694. doi: 10.1073/pnas.1002720107

Garrett-Bakelman, F. E., Sheridan, C. K., Kacmarczyk, T. J., Ishii, J., Betel, D., Alonso, A., et al. (2015). Enhanced reduced representation bisulfite sequencing for assessment of DNA methylation at base pair resolution. J. Vis. Exp. 96:e52246. doi: 10.3791/52246

Gaudet, F., Hodgson, J. G., Eden, A., Jackson-Grusby, L., Dausman, J., Gray, J. W., et al. (2003). Induction of tumors in mice by genomic hypomethylation. Science 300, 489-492. doi: 10.1126/science.1083558

Globisch, D., Munzel, M., Muller, M., Michalakis, S., Wagner, M., Koch, S., et al. (2010). Tissue distribution of 5-hydroxymethylcytosine and search for active demethylation intermediates. PLOS ONE 5:e15367. doi: 10.1371/journal.pone.0015367

Goessling, W., and Sadler, K. C. (2015). Zebrafish: an important tool for liver disease research. Gastroenterology 149, 1361-1377. doi: 10.1053/j.gastro.2015.08.034

Gu, H., Smith, Z. D., Bock, C., Boyle, P., Gnirke, A., and Meissner, A. (2011). Preparation of reduced representation bisulfite sequencing libraries for genome-scale DNA methylation profiling. Nat. Protoc. 6, 468-481. doi: 10.1038/nprot.2010.190

Gu, J., Stevens, M., Xing, X., Li, D., Zhang, B., Payton, J. E., et al. (2016). Mapping of variable DNA methylation across multiple cell types defines a dynamic regulatory landscape of the human genome. G3 (Bethesda) 6, 973-986. doi: $10.1534 / g 3.115 .025437$
Hagemann, S., and Hammer, S. E. (2006). The implications of DNA transposons in the evolution of P elements in zebrafish (Danio rerio). Genomics 88, 572-579. doi: 10.1016/j.ygeno.2006.06.010

Hartung, T., Zhang, L., Kanwar, R., Khrebtukova, I., Reinhardt, M., Wang, C., et al. (2012). Diametrically opposite methylome-transcriptome relationships in highand low-CpG promoter genes in postmitotic neural rat tissue. Epigenetics 7, 421-428. doi: 10.4161/epi.19565

Howard, G., Eiges, R., Gaudet, F., Jaenisch, R., and Eden, A. (2008). Activation and transposition of endogenous retroviral elements in hypomethylation induced tumors in mice. Oncogene 27, 404-408. doi: 10.1038/sj.onc.1210631

Illingworth, R. S., and Bird, A. P. (2009). CpG islands - "A rough guide”. FEBS Lett. 583, 1713-1720. doi: 10.1016/j.febslet.2009.04.012

Irizarry, A. R. (2009). Genome-wide methylation analysis of human colon cancer reveals similar hypo- and hypermethylation at conserved tissue- specific CpG island shores. Nat. Genet. 41, 178-186. doi: 10.1038/ng.298.Genome-wide

Irizarry, R. A., Ladd-Acosta, C., Wen, B., Wu, Z., Montano, C., Onyango, P., et al. (2009). The human colon cancer methylome shows similar hypo- and hypermethylation at conserved tissue-specific CpG island shores. Nat. Genet 41, 178-186. doi: 10.1038/ng.298

Jacob, V., Chernyavskaya, Y., Chen, X., Tan, P. S., Kent, B., Hoshida, Y., et al. (2015). DNA hypomethylation induces a DNA replication-associated cell cycle arrest to block hepatic outgrowth in uhrf1 mutant zebrafish embryos. Development 142, 510-521. doi: 10.1242/dev.115980

Kapusta, A., Kronenberg, Z., Lynch, V. J., Zhuo, X., Ramsay, L., Bourque, G., et al. (2013). Transposable elements are major contributors to the origin, diversification, and regulation of vertebrate long noncoding RNAs. PLoS Genet. 9:e1003470. doi: 10.1371/journal.pgen.1003470

Kim, D., Pertea, G., Trapnell, C., Pimentel, H., Kelley, R., and Salzberg, S. L. (2013). TopHat2: accurate alignment of transcriptomes in the presence of insertions, deletions and gene fusions. Genome Biol. 14:R36. doi: 10.1186/gb-201314-4-r36

Lam, S. H., Wu, Y. L., Vega, V. B., Miller, L. D., Spitsbergen, J., Tong, Y., et al. (2006). Conservation of gene expression signatures between zebrafish and human liver tumors and tumor progression. Nat. Biotechnol. 24, 73-75. doi: 10.1038/nbt1169

Lam, W. L., Lee, T. S., and Gilbert, W. (1996). Active transposition in zebrafish. Proc. Natl. Acad. Sci. U.S.A. 93, 10870-10875. doi: 10.1073/pnas.93.20.10870

Lee, H. J., Lowdon, R. F., Maricque, B., Zhang, B., Stevens, M., Li, D., et al. (2015). Developmental enhancers revealed by extensive DNA methylome maps of zebrafish early embryos. Nat. Commun. 6, 6315. doi: 10.1038/ncomms7315

Lee, T.-F., Zhai, J., and Meyers, B. C. (2010). Conservation and divergence in eukaryotic DNA methylation. Proc. Natl. Acad. Sci. U.S.A. 107, 9027-9028. doi: 10.1073/pnas.1005440107

Lee, Y. K., Jin, S., Duan, S., Lim, Y. C., Ng, D. P., Lin, X. M., et al. (2014). Improved reduced representation bisulfite sequencing for epigenomic profiling of clinical samples. Biol. Proced. Online 16, 1. doi: 10.1186/1480-9222-16-1

Li, E., Beard, C., and Jaenisch, R. (1993). Role for DNA methylation in genomic imprinting. Nature 366, 362-365. doi: 10.1038/366362a0

Long, H. K., King, H. W., Patient, R. K., Odom, D. T., and Klose, R. J. (2016). Protection of CpG islands from DNA methylation is DNA-encoded and evolutionarily conserved. Nucleic Acids Res. gkw258. doi: 10.1093/nar/gkw258

Long, H. K., Sims, D., Heger, A., Blackledge, N. P., Kutter, C., Wright, M. L., et al. (2013). Epigenetic conservation at gene regulatory elements revealed by non-methylated DNA profiling in seven vertebrates. Elife 2, 1-19. doi: 10.7554/eLife.00348

McGhee, J. D., and Ginder, G. D. (1979). Specific DNA methylation sites in the vicinity of the chicken $\beta$-globin genes. Nature 280, 419-420. doi: 10.1038/280419a0

Meissner, A., Mikkelsen, T. S., Gu, H., Wernig, M., Hanna, J., Sivachenko, A., et al. (2008). Genome-scale DNA methylation maps of pluripotent and differentiated cells. Nature 454, 766-770. doi: 10.1038/nature07107

Messerschmidt, D. M., Knowles, B. B., and Solter, D. (2014). DNA methylation dynamics during epigenetic reprogramming in the germline and preimplantation embryos. Genes Dev. 28, 812-828. doi: 10.1101/gad.234294.113.process

Mirbahai, L., Williams, T. D., Zhan, H., Gong, Z., and Chipman, J. K. (2011). Comprehensive profiling of zebrafish hepatic proximal promoter CpG 
island methylation and its modification during chemical carcinogenesis. BMC Genomics 12:3. doi: 10.1186/1471-2164-12-3

Mudbhary, R., Hoshida, Y., Chernyavskaya, Y., Jacob, V., Villanueva, A., Fiel, M. I., et al. (2014). UHRF1 overexpression drives DNA hypomethylation and hepatocellular carcinoma. Cancer Cell 25, 196-209. doi: 10.1016/j.ccr.2014.01.003

Potok, M. E., Nix, D. A., Parnell, T. J., and Cairns, B. R. (2013). Reprogramming the maternal zebrafish genome after fertilization to match the paternal methylation pattern. Cell 153, 759-772. doi: 10.1016/j.cell.2013.04.030

Reid, D. S. (2010). Taking the measure of the methylome. Nat. Biotechnol. 28, 199-218. doi: 10.1002/9780470958193.ch16

Sadler, K. C., Krahn, K. N., Gaur, N. A., and Ukomadu, C. (2007). Liver growth in the embryo and during liver regeneration in zebrafish requires the cell cycle regulator, uhrf1. Proc. Natl. Acad. Sci. U.S.A. 104, 1570-1575. doi: 10.1073/pnas.0610774104

Singer, Z. S., Yong, J., Tischler, J., Hackett, J. A., Altinok, A., Surani, M. A., et al. (2014). Dynamic heterogeneity and DNA methylation in embryonic stem cells. Mol. Cell 55, 319-331. doi: 10.1016/j.molcel.2014.06.029

Slotkin, R. K., and Martienssen, R. (2007). Transposable elements and the epigenetic regulation of the genome. Nat. Rev. Genet. 8, 272-285. doi: $10.1038 /$ nrg2072

Smith, S. S., and Crocitto, L. (1999). DNA methylation in eukaryotic chromosome stability revisited: DNA methyltransferase in the management of DNA conformation space. Mol. Carcinog. 26, 1-9. doi: 10.1002/(SICI)10982744(199909)26:1 <1::AID-MC1 > 3.0.CO;2-P

Smith, Z. D., Gu, H., Bock, C., Gnirke, A., and Meissner, A. (2009). Highthroughput bisulfite sequencing in mammalian genomes. Methods 48, 226-232. doi: 10.1016/j.ymeth.2009.05.003

Spiers, H., Hannon, E., Schalkwyk, L. C., Smith, R., Wong, C. C. Y., Donovan, M. C. O., et al. (2015). Methylomic trajectories across human fetal brain development. Genome Res. 25, 338-352. doi: 10.1101/gr.180273.114

Trapnell, C., Roberts, A., Goff, L., Pertea, G., Kim, D., Kelley, D. R., et al. (2012). Differential gene and transcript expression analysis of RNA-seq experiments with TopHat and Cufflinks. Nat. Protoc. 7, 562-578. doi: 10.1038/nprot.2012.016

Xi, Y., and Li, W. (2009). BSMAP: whole genome bisulfite sequence MAPping program. BMC Bioinformatics 10:232. doi: 10.1186/1471-2105-10-232
Yoder, J. A., Walsh, C. P., and Bestor, T. H. (1997). Cytosine methylation and the ecology of intragenomic parasites. Trends Genet. 13, 335-340. doi: 10.1016/S0168-9525(97)01181-5

Zemach, A., McDaniel, I. E., Silva, P., and Zilberman, D. (2010). Genome-wide evolutionary analysis of eukaryotic DNA methylation. Science 328, 916-919. doi: 10.1126/science.1186366

Zeng, J., Konopka, G., Hunt, B. G., Preuss, T. M., Geschwind, D., and Yi, S. V. (2012). Divergent whole-genome methylation maps of human and chimpanzee brains reveal epigenetic basis of human regulatory evolution. Am. J. Hum. Genet. 91, 455-465. doi: 10.1016/j.ajhg.2012.07.024

Zhang, Y., Wang, H., Zhou, D., Moody, L., Lezmi, S., Chen, H., et al. (2015). Highfat diet caused widespread epigenomic differences on hepatic methylome in rat. Physiol. Genomics 47, 514-523. doi: 10.1152/physiolgenomics.00110.2014

Zhao, L., Sun, M., Li, Z., Bai, X., Yu, M., Wang, M., et al. (2014). The dynamics of DNA methylation fidelity during mouse embryonic stem cell self-renewal and differentiation. Genome Res. 24, 1296-1307. doi: 10.1101/gr.163147.113

Zheng-Bradley, X., Rung, J., Parkinson, H., and Brazma, A. (2010). Large scale comparison of global gene expression patterns in human and mouse. Genome Biol. 11:R124. doi: 10.1186/gb-2010-11-12-r124

Zhou, X., Li, D., Lowdon, R. F., Costello, J. F., and Wang, T. (2014). MethylC track: visual integration of single-base resolution DNA methylation data on the WashU EpiGenome Browser. Bioinformatics 30, 2206-2207. doi: 10.1093/bioinformatics/btu191

Ziller, M. J., Müller, F., Liao, J., Zhang, Y., Gu, H., Bock, C., et al. (2011). Genomic distribution and Inter-Sample variation of Non-CpG methylation across human cell types. PLoS Genet. 7:e1002389. doi: 10.1371/journal.pgen.1002389

Conflict of Interest Statement: The authors declare that the research was conducted in the absence of any commercial or financial relationships that could be construed as a potential conflict of interest.

Copyright (c) 2016 Zhang, Hoshida and Sadler. This is an open-access article distributed under the terms of the Creative Commons Attribution License (CC BY). The use, distribution or reproduction in other forums is permitted, provided the original author(s) or licensor are credited and that the original publication in this journal is cited, in accordance with accepted academic practice. No use, distribution or reproduction is permitted which does not comply with these terms. 